\title{
ACERCA DE INCONMENSURABILIDAD Y TRADUCCIÓN
}

Elizabeth Padilla UNCO

\section{I}

Para responder a las críticas realizadas a la tesis de la inconmensurabilidad entre teorías científicas sucesivas, que sostiene la imposibilidad de pretender compararlas una vez aceptada la inconmensurabilidad de las mismas, Kuhn propone la estrategia de distinguir entre interpretación y traducción en toda traducción real. La crítica afirma que si la tesis defiende la inconmensurabilidad de las teorías, no podrían plantearse diferencias ni comparaciones entre las mismas, pues esto implica la inexistencia de un lenguaje común que permita relacionarlas. El asunto es que, por otra parte, como Kuhn sostiene, de hecho vienen realizándose reconstrucciones exitosas de textos científicos antiguos sin reparar, aparentemente, en la necesidad de contar con un lenguaje común, a menos que tales reconstrucciones no supongan procesos exclusivamente traductivos. Al afirmar la distinción entre ambos términos adjudica el de traducción a la tradición analítica, la cual propone que este proceso puede entenderse en términos puramente referenciales, mientras que la interpretación es el proceso mediante el cual se da cuenta de la intensionalidad de los términos a través de la elección de aspectos que parecieran prescindir de un lenguaje en común. Según Kuhn, entonces, las críticas antes señaladas son deudoras de la tradición analítica que ha confundido estos dos procesos que se dan en toda traducción real. Al respecto 
afirma que tal confusión es posible rastrearla ya en Palabra y $\mathrm{Ob}$ jeto de Quine, para lo cual revisa críticamente su conocido manual de traducción. Una de las causas de esta confusión, según Kuhn, es el rechazo por parte de Quine de los requisitos intensionales que debe cumplir una traducción adecuada. Para Kuhn una traducción perfecta preservaría estas intensionalidades y el hecho de que no se logren no nos exime de intentar aproximarnos a este ideal.

Es así que me propongo revisar si es que puede atribuírsele al Quine de Palabra y Objeto la confusión señalada para evaluar a partir de ello la pertinencia de las características que Kuhn le atribuye a la interpretación como reaseguro de la inconmensurabilidad en su version local.

\section{II}

En primer término veamos los problemas a los que se ve enfrentado Kuhn en su defensa de la inconmensurabilidad. Con el término inconmensurabilidad Kuhn va a designar la imposibilidad de expresar todos los términos de una teoría en el vocabulario de la otra ${ }^{(1)}$. Decir que dos teorías son inconmensurables es afirmar que son intraducibles, es decir, no hay ningún lenguaje neutral, ni de otro tipo, al que ambas puedan traducirse, sin resto o pérdida de significado. No obstante, esto no significa que no se puedan comparar, como objetan sus críticos. Entre estos podemos mencionar a Putnam, ya que sobre sus críticas giran las respuestas dadas por Kuhn a los detractores de la inconmensurabilidad.

Las mismas pueden parafrasearse del siguiente modo: afirmar que dos teorías son inconmensurables supone que estén

(1) Si bien Kuhn amplía el uso de este término abarcando con él, además del lenguaje, diferencias en los métodos y normas de resolución, es claro que el tratamiento del tema lo evalúa en términos lingüísticos.Cfr. Kuhn, T. [1987] 
formuladas en lenguajes intraducibles; de lo cual debería concluirse: a) no pueden compararse y ningún argumento basado en la evidencia puede ser relevante para la elección entre teorías. b) Es imposible traducir teorías antiguas al lenguaje moderno. Ahora bien, dado que es precisamente esto lo que Kuhn ha venido realizando, la pregunta de fondo es qué entiende este autor cuando habla de inconmensurabilidad.

Para responder a las críticas el autor va a señalar en una primera instancia la procedencia del término, con el objeto de aclarar que desde lo terminológico "inconmensurabilidad" no está reñido con "comparabilidad". El término inconmensurabilidad proviene de las matemáticas. En un triángulo rectángulo isósceles la hipotenusa es inconmensurable con su lado, o la circunferencia de un círculo con su radio. Esto significa que no hay una unidad de longitud contenida un número entero de veces, "no hay medida común" [Kuhn 1987,99]. No obstante, podemos comparar ambos miembros del par con algún grado de aproximación. Esto es posible porque las técnicas geométricas se aplican a ambos miembros del par inconmensurable.

Si nos trasladamos al vocabulario conceptual de una teoría científica y su contexto, el término inconmensurabilidad funciona metafóricamente, "no hay medida común" se convierte en "sin lenguaje común". Pero en ningún momento la inconmensurabilidad, en su sentido metafórico o literal, supone incomparabilidad. Surgen, según Kuhn, problemas de traducción sólo con un pequeño subgrupo de términos, que en la mayoría de los casos se interdefinen, y con los enunciados que los contienen; el resto de los términos al ser comunes a ambas teorías son traducidos en forma homófona. Kuhn llamará a esta versión más modesta de inconmensurabilidad «inconmensurabilidad local» [Kuhn 1987, 100]. 
Ahora bien, Kuhn en la versión local afirma que la mayoría de los términos de las teorías conservan sus significados, constituyéndose en base suficiente para discutir las diferencias y las comparaciones relevantes en la elección de teorías, que surgen a partir del pequeño subgrupo de términos intraducibles. Es así como, tales comparaciones se sustentan en la presuposición de que hay términos que conservan sus significados a través de los cambios de teorías sin verse afectados por aquellos que sí se modifican. Recordemos que este supuesto permite restringir la inconmensurabilidad al nivel local, asegurándose de este modo la comparabilidad. Con respecto a esto último, el autor reconoce que su versión local de inconmensurabilidad es discutible, ya que en el estado actual de la teoría del significado la diferencia entre téminos que cambian su significado y aquellos que lo conservan es difícil de explicar o aplicar. De ahí la importancia de ofrecer una alternativa al problema de la inconmensurabilidad y su relación con la “invariancia de significado" [Kuhn 1987, 101].

El tratamiento de la segunda objeción (la de la historia), que sobrevive a la versión local, permitirá la búsqueda de tal alternativa. Sin embargo, el carácter polémico de la "invariancia del significado", problema que conlleva toda traducción, lleva a Kuhn a considerar que toda traducción real encierra dos procesos distinguibles, traducción e interpretación. Recordemos que la segunda objeción afirma que, de acuerdo con la inconmensurabilidad -en su versión local- es imposible traducir teorías antiguas al lenguaje moderno, sin embargo es lo que vienen haciendo con éxito historiadores (el mismo Kuhn) y antropólogos. Los supuestos que hacen posible estas traducciones son fundamentales en los argumentos dirigidos contra la inconmensurabilidad y sustentan las principales críticas realizadas a este autor, pues afirman que el éxito de este proceso es incompatible con la inconmensurabilidad, 
aún en su versión local. No obstante, según Kuhn, éstas críticas incurren en el defecto de describir los resultados de la interpretación como una traducción -advirtiéndose ya esta confusión, como señalamos, en Palabra y Objeto de Quine- Según Kunh dos teorías localmente inconmensurables y por consiguiente intraducibles se pueden comparar mediante un proceso que involucre interpretación, ya que la interpretación no supone traducibilidad. De allí la necesidad de no confundir, como lo haría la tradición analítica, interpretación con traducción. Para el autor, el que pueda efectuarse la interpretación de un texto en una lengua nueva o en una versión antigua de la propia no implica traducción, manteniéndose la inconmensurabilidad local. Al respecto afirma: "Yo mantengo que la interpretación -...- es distinta de la traducción... La confusión es fácil porque la traducción real contiene a menudo, o quizá siempre, al menos un pequeño componente interpretativo. Pero en este caso es necesario considerar que la traducción real encierra dos procesos distinguibles». [Kuhn 1987, 102-103]. En cuanto al modo en que entiende Kuhn el proceso de traducción, si bien advierte que éste dista mucho de acercarse a una traducción real, no obstante afirma que dichas características "derivan directamente... de la naturaleza y función de un manual de traducción quineano". [Kuhn 1987, 104].

La traducción es algo que realiza una persona que sabe dos idiomas. La tarea del traductor consiste en reemplazar palabras o secuencias de palabras en el texto por palabras o secuencias de palabras en el otro idioma, con el objeto de producir un texto equivalente. En cuanto a qué significa producir un texto equivalente, Kuhn afirma: «... el texto traducido cuenta más o menos la misma historia, ...presenta más o menos las mismas ideas, 0 ...describe más o menos la misma situación que el texto del cual es una traducción». [Kuhn 1987, 103]. Esta caracterización resulta suma- 
mente vaga, ya que el producir un texto equivalente está sujeto a preservar "más o menos", entre otras cosas, la misma historia o las mismas ideas o la misma situación del texto original. Pero entiendo que es de este modo como Kuhn logra eludir referirse al tema del significado y su invariancia, sin verse demasiado comprometido a dar una respuesta a un problema que él no logra resolver. Al respecto afirma en relación con la inconmensurabilidad “...que significado no es el mejor encabezamiento para una discusión sobre inconmensurabilidad. Sin embargo, no tenemos hoy en día ninguna alternativa más adecuada." [Kuhn 1987, 101] por lo cual el recurso a la interpretación resultaría una alternativa a ese problema, dado que precisamente permite prescindir de la "invariancia del significado"

Para llevar a cabo una interpretación la persona que la efectúa puede inicialmente conocer sólo una lengua. Es así que para Kuhn el "traductor radical", que aparece en Palabra y Objeto de Quine, es alguien que se encuentra en una situación de interpretación, más que de traducción, ya que se parte del conocimiento de la propia lengua de la que se es hablante para catapultarse a otra. El texto sobre el que trabaja el traductor radical parece consistir al principio en un conjunto de ruidos o inscripciones ininteligibles. De ahí que a los fines de arribar a una interpretación tendrá en cuenta la conducta y las circunstancias que rodean la producción del discurso, suponiendo en todo momento que se puede extraer un sentido de la conducta lingüística de aquellos que son observados (principio de caridad). Acorde a esos elementos elaborará hipótesis que hagan inteligibles las expresiones lingüísticas investigadas. Si esta empresa tiene éxito habrá aprendido una nueva lengua o una versión más antigua de la propia. Ahora bien, que esta lengua pueda traducirse a aquella con la cual él comenzó es toda una cuestión. Siguiendo a Kuhn, "Aprender una nueva lengua no 
es lo mismo que traducir de ella a la propia. Tener éxito en lo primero no implica necesariamente que también se vaya a obtener éxito en lo segundo". [Kuhn 1987, 105].

La descripción que acabamos de realizar sigue, para aquellos que conocen a Quine, fielmente el manual de traducción. Sin embargo, ¿cuál es la índole de la diferencia para que Kuhn nos proponga entender este proceso como interpretación? Ambos autores entienden que la traducción supone conservación del significado, no obstante ambos reconocen también que tal pretensión es una cuestión problemática. Ahora bien, mientras que para Quine lo que se preserva en una traducción adecuada es el significado estimulativo, para Kuhn el elegir la sustitución del término traducción por interpretación lo conduce a no aceptar todas las consecuencias del manual quineano y por ende a caer en el tema de la referencia, recurso del que ya Quine había mostrado su inescrutabilidad.

Veamos cómo ocurre esto. Todo lo afirmado hasta ahora tiene como objetivo mostrar de qué manera Kuhn defiende la inconmensurabilidad local frente a los embates de los que consideran que sostener inconmensurabilidad y comparabilidad es incurrir en franca contradicción. Sin embargo observo que una vez criticada la supuesta confusión en la que incurre la tradición analítica en general y Quine en particular, Kuhn no logra precisar características propias atribuibles a la interpretación, aclarándonos, simplemente, que esta no requiere de un lenguaje en común.

Con respecto a la confusión que Kuhn le adjudica a Quine en relación a la interpretación y la traducción, entiendo que Quine no plantea tal distinción pues sus objetivos son muy otros al describir la situación de traducción radical. El interés de Quine en Palabra y Objeto consiste en revisar el concepto mismo de signifi- 
cado para, una vez abandonados los supuestos mentalistas de significado y la distinción analítico/sintético, tratar de construir una noción aceptable del mismo. Al respecto afirma en La relatividad ontológica y otros ensayos: "Mis observaciones sobre la indeterminación comenzaron como un reto a la igualdad de significado... Ciertamente, la igualdad de significado es una noción oscura, repetidamente atacada". [Quine 1969, 53]

Abandonada la concepción mentalista Quine intenta construir el concepto de significado a través de la elucidación de los criterios de una traducción aceptable. En cuanto a qué sean los significados, Quine afirma: “...la significación es lo que una sentencia tiene en común con su traducción..." [Quine 1960, 45]. Estudiar esta cuestión entre lenguas que ya poseen manuales de traducción resulta poco esclarecedor, por ello afirma: "La traducción entre lenguajes emparentados,..., cuenta con la ayuda del parecido entre formas verbales y también emparentadas... El caso relevante para nuestros fines es empero el de la traducción radical, esto es, la traducción del lenguaje de un pueblo al que se llega por vez primera". [Quine 1960, 41].

Dijimos anteriormente que la significación es lo que tienen en común la sentencia con su traducción. "Lo que tienen en común", en el caso de Quine, serán ciertas disposiciones a la conducta lingüística. Las disposiciones conectan estímulos sensibles con respuestas lingüísticas de asentimiento y disentimiento. Esto nos permite definir la noción quineana de significado estimulativo afirmativo como "...la clase de todas las estimulaciones (...) que provocarían asentimiento" [Quine 1960,45], del mismo modo para significado estimulativo negativo sustituyendo "asentir" por "disentir". Los significados estimulativos son disposiciones a la conducta observable (asentimientos y disentimientos) en circunstancias manifiestas. Por ejemplo: "pasa un conejo, el indígena dice 
"Gavagai" y el lingüista anota la sentencia "Conejo" como traducción provisional..." [Quine 1960, 41] ${ }^{(2)}$.

Una vez presentada esta descripción sumaria de los aspectos que considero más relevantes del manual de traducción quineano estamos en condiciones de evaluar la pertinencia de las críticas que realiza Kuhn a los ejemplos dados por Quine, como así también a las alternativas que propone.

Como se dijo anteriormente para Kuhn interpretar una lengua nueva no supone traducción. De allí que para este autor, Quine en su manual de traducción confunde ambos procesos; un ejemplo de esto sería la traducción que propone Quine de la sentencia "Gavagai". Con respecto a "Gavagai", Kuhn sugiere que en lugar de traducir el término es posible aprender a usarlo al modo en que lo hacen los indígenas a partir de reconocer el animal al cual refiere el término; de este modo se evitaría el requisito de la previa familiaridad del traductor con el objeto a traducir. Sin embargo observamos que Kuhn no tiene en cuenta que lo que provoca el asentimiento ante la pregunta "¿Gavagai?" son estimulaciones compartidas por el traductor y el indígena y no el animal al que refiere la expresión que es de exclusivo conocimiento de los nativos. Según Quine lo que el traductor ha de hacer en la traducción de sentencias es correlacionar oraciones nativas con oraciones de su lenguaje que tienen el mismo significado estimulativo. Por otra parte, en lo que hace al requisito de familiaridad mencionado cabe destacar que Quine relativiza la aparente necesidad del mismo pues lo que importa para el caso es recoger el rango de las estimulaciones significativas compartidas; es decir, parafraseando a Quine, la significación estimulativa es la realidad objetiva que el lingüista tiene que indagar cuando se dedica a tareas de traducción radical. La

(2) Quine utiliza mayúsculas para referirse a sentencias y minúsculas para términos 
significación estimulativa de una sentencia como "Gavagai" es el conjunto de disposiciones actuales, presentes en el indígena, por las cuales asentirá a o discrepará de la sentencia; y esto es precisamente lo que el lingüista debe evaluar.

No obstante, la observación de Kuhn se puede entender si se hace lugar a las exigencias propias a las que se ve enfrentado como historiador de la ciencia en su intento de reconstruir el sentido de los textos científicos antiguos, a diferencia del lingüista quineano que en situación de traducción radical intenta confeccionar un manual de traducción de una lengua que no guarda ninguna relación con la propia. Quiero decir, es cierto que para el primero la tarea de apelar a una situación estimulativa compartida de sentencias observacionales parecería imposible. Sin embargo, no es este un asunto al que debamos hacerle lugar en este momento, ya que por otra parte el mismo Kuhn pareciera no considerarlo en su discusión.

Como acabamos de ver la propuesta de Kuhn para "Gavagai" consiste en aprender el uso de este término tal como lo hacen los nativos. La dificultad de esto consiste en que produce la modificación de la lengua del hablante por incorporación de términos propios de la lengua nativa. Por otra parte el resultado de esta actividad no podría ser calificada de traducción. Es así que Kuhn ofrece otra alternativa a los fines de la traducción: intentar describir los referentes del término "gavagai". Si la descripción se adecua a todas las criaturas que provocan la proferencia "gavagai", entonces podemos afirmar que hemos dado con la traducción buscada. Aquí, por lo tanto, no surgen problemas de intraducibilidad; en palabras de Kuhn, de inconmensurabilidad.

Ahora bien, Quine, al abandonar la concepción mentalista del significado y asumir una posición conductista, sostiene que el 
significado de las sentencias observacionales consiste en el significado estimulativo y este no supone referencialidad. De ahí que la traducción de la sentencia observacional "Gavagai" se la puede hacer corresponder con "Conejo" o, "Mirad, un conejo", pero esto no significa que el término "gavagai" refiera al témino "conejo" es decir, en palabras de Quine, ambos términos no son coextensivos (verdaderos de las mismas cosas). Al respecto Quine afirma: "Consideremos, en efecto, "gavagai". ¿Quién sabe si los objetos a los que se aplica ese término no son en última instancia conejos, sino meros estadios o breves segmentos temporales de conejos? En cualquier caso, las situaciones estimulativas que provocan el asentimiento a "Gavagai" lo provocarían también a "Conejo"..." [Quine $1960,64]$. Por lo tanto, cuando el lingüista concluye que un "gavagai" es un conejo partiendo de la misma significación estimulativa de "Gavagai" y "Conejo", está suponiendo que el nativo es parecido a nosotros en la medida en que posee un término general breve para designar conejos y ningún término general $\mathrm{y}$ breve para designar estadios o partes de conejos. Si nuestra duda consiste en si acaso esto se debe a un defecto especial en la formulación de la significación estimulativa, que de últimas se resolvería con más ostensión, Quine responde: "Nada que no se distinga ya en la significación estimulativa puede distinguirse por ostensión...". [Quine 1960, 66]. A lo que agrega, a menos que se hagan preguntas sobre identidad y diversidad. Pero poder realizar estas preguntas supone un conocimiento profundo de la lengua nativa, es más, haber decidido qué expedientes nativos tratan acerca de la referencia objetiva.

De acuerdo a lo afirmado no es posible, como intenta Kuhn, obtener la traducción de ningún término mediante la descripción de todos sus referentes. Pues con lo que el lingüista cuenta al iniciar la traducción es con hipótesis acerca de situaciones estimulativas compartidas y no otra cosa. La referencia va a que- 
dar fijada una vez que hayamos confeccionado nuestro manual de traducción, es decir, va a depender de las hipótesis analíticas que hayamos seleccionado a la hora de confeccionarlo. Y la posibilidad de elegir entre distintos sistemas de hipótesis analíticas nos remite a la indeterminación de la traducción; según palabras de Quine:"... sistemas rivales de hipótesis analíticas pueden recoger todas las disposiciones lingüísticas de cada uno de los lenguajes considerados y arrojar sin embargo en innumerables casos traducciones totalmente dispares.." [Quine 1960, 86] De ahí que no podamos llevar a cabo la alternativa que propone Kuhn de ofrecer una descripción adecuada de todos los objetos ante los cuales los hablantes profieren el término "gavagai" [Kuhn 1987, 106].

Como podemos observar, pareciera que Kuhn reduce los procesos traductivos que operan en la traducción real a aspectos puramente referenciales -caracterización que adjudica a los analíticos-, mientras que, como acabamos de mostrar, en Quine la traducibilidad no se identifica con determinación de la referencialidad de los términos. Sin embargo también vimos que para Kuhn hay ciertos términos intraducibles, para los cuales propone tratarlos según relaciones de uso mediante procesos de aprendizaje de otra lengua. Kuhn entiende que la intraducibilidad de esos términos se debe al modo en que los indígenas estructuran el mundo animal, diferente al modo en que lo hacen los hablantes castellanos. En ese caso no podríamos traducir el término, pero sí aprender a usarlo y cuando lo hacemos estamos hablando la lengua indígena. Esto se trata, según Kuhn, de un caso de inconmensurabilidad. De este modo, el problema de la inconmensurabilidad de ciertos términos se relacionaría con el tema de la inescrutabilidad de la referencia.

Recordemos que siguiendo a Kuhn podríamos siempre comparar los éxitos y fracasos de dos teorías que, aún siendo in- 
conmensurables, como ya señalamos, se las puede comparar a partir de términos que preservan sus significados, para llegar así a tomar decisiones respecto de cual de ambas teorías elegir. Es claro que en principio Kuhn se deja seducir por el carácter homófono de esos términos. El problema, por supuesto es suponer a partir de ello un mismo sostén referencial para ambos, sobre todo si a la vez partimos de teorías que estructuran el mundo de manera distinta, según señala el mismo Kuhn. Por otra parte, Quine afirma al respecto que la indeterminación de la traducción no es un problema que afecta sólo al traductor radical sino que empieza en nuestra propia lengua, nuestra propia ontología está indeterminada y es inescrutable; en otras palabras, la indeterminación afecta por igual a los hablantes de una misma lengua, es más, llevado a su extremo afecta también al uso que un sujeto hace de sus proferencias en distintos momentos de su vida. De ahí que los términos que parecen permanecer invariantes a través del cambio de teorías no nos proporcionan, como esperaba Kuhn, una base segura para establecer la comparabilidad. Kuhn va a corregir más adelante esta presunción, ante el estado de discusión de las teorías del significado, evitando totalmente hablar de "significado"; proponiéndose en cambio "... discutir en términos generales y casi metafísicos, cómo identifican los miembros de una comunidad lingüística los referentes de los términos que emplean." [Kuhn 1987, 127]. Aquí se va a preguntar fundamentalmente cómo puede ser que personas con criterios diferentes identifican los mismos referentes para sus términos. La cuestión radica en qué significa "identificar los mismos referentes para sus términos", ya que -como podemos advertir desde Quine- cuando me comunico con otro individuo que comparte mi lenguaje, parto de la hipótesis de que con las mismas palabras ese individuo se refiere a lo mismo que yo; pero esa hipótesis nunca puede ser plenamente confirmada, pues únicamente dispongo de las conductas lingüísticas que, como sabemos, resul- 
tan insuficientes para determinar la referencia de la expresión. Esto se debe a que el traductor no puede inferir con certeza, a partir de la conducta verbal, los criterios de individuación que éste emplea para indentificar las cosas del mundo ${ }^{(3)}$. Por lo tanto la referencia de un término viene determinada por su relación con otros términos, dentro de cierto marco lingüístico. Si cambiamos de marco lingüístico cambiaremos de referencia, dado que no existe un punto de vista supralingüístico absoluto desde el cual poder fijar la referencia de cada término con independencia de sus relaciones con otros términos y desde ésta atalaya poder comparar teorías inconmensurables.

\section{III}

Para finalizar voy a evaluar si esta disputa acaso puede ser dirimida entendiéndola como una mera diferencia de objetivos en las tareas que emprenden ambos autores. En este sentido, Quine, como ya fue dicho, investigará acerca de cómo las irritaciones de la superficie sensible engendran, a través del lenguaje, el conocimiento del mundo. Para esto trata de establecer qué parte del lenguaje puede recibir significado evaluándola sobre la base de las condiciones de estimulación, prescidiendo para ello de elementos interpretativos o intensionales. En el caso de Kuhn hay un propósito estratégico: cómo superar las críticas a la tesis de la inconmensurabilidad en su versión local. Para ello establece la distinción de dos procesos que se dan en la traducción: traducción e interpretación, pero dadas las dificultades que plantea el supuesto de la invariancia del significado, objetivo que persigue toda traducción, recurre al expediente intensionalista. Este último se re-

(3) Cfr. Quine 1969, págs. 49-52. 
laciona, de alguna manera, con los usos lingüísticos que hacen los miembros de una comunidad de sus términos, a los cuales se apelaría en la reconstrucción de los textos científicos.

El tema es si con lo mencionado logra satisfacer los requerimientos necesarios para seguir sosteniendo la tesis de la inconmensurabilidad. Mi evaluación al respecto es que -sin pretender por ello expedirme sobre los alcances y/o virtudes de esta tesis en tanto tratamiento epistémico de teorías sucesivas-, Kuhn no ha logrado brindar una respuesta satisfactoria a las críticas, como parece quedar claro a la luz de lo que hemos expuesto. La debilidad de sus argumentos no reside tan sólo en la atribución de confusión a Quine donde creo haber mostrado que no la hay, sino también en que su propuesta supone la reducción de todo proceso traductivo y aún de los interpretativos -si es que hacemos lugar a su distinción- a aspectos puramente referenciales. Seguramente esto se debe a que no ha interpretado cabalmente el sentido de la traducción radical ofrecido por Quine en su manual de traducción. Como vimos, según Quine, la referencia puede ser fijada a partir de cada manual de traducción. En Kuhn, no está clara su posición al respecto sino más bien hay una situación pendular que por momentos nos invita a pensar en un sentido fuerte de referencia, entendida como lo que el significado de los términos debe preservar a partir de la adaptación del lenguaje al mundo y en otros la referencia como la que nos debe conducir a los significados de los términos. Es interesante observar que un modo de resolver tal situación lo brinda precisamente algo que no recoge Kuhn, como es la noción de significación estimulativa de enunciados observacionales introducida por el manual de traducción quineano; la cual aporta con esto un elemento considerable en favor del intento kuhniano de la búsqueda de un lenguaje en la zona no localmente inconmensurable que permita la comparación de teorías inconmensurables. 


\section{RESUMEN}

Kuhn propone en "Conmensurabilidad, comparabilidad y comunicabilidad" la estrategia de distinguir entre interpretación y traducción para responder a las críticas realizadas a su tesis de la inconmensurabilidad entre teorías científicas sucesivas. Esto lo lleva a considerar que la tradición analítica -ejemplificada en Quine de Palabra y Objeto- confunde ambos procesos. Me propongo revisar si es que puede atribuírsele a este autor la confusión señalada, para evaluar a partir de ello la pertinencia de las características que Kuhn le atribuye a la interpretación como reaseguro de la inconmensurabilidad en su version local.

\section{BIBLIOGRAFIA}

Kuhn,T., [1987] What are Scientific Revolutions?, Cambridge-Londres, Massachusetts Institute of Technology, 1987 (las citas corresponden a la versión castellana ¿Qué son las revoluciones cientificas? y otros ensayos, Barcelona, Paidós, 1989)

Quine, W.V. [1969] Ontological Relativity and Other Essays, Nueva york, Columbia University Press (las citas corresponden a la versión castellana La relatividad ontológica y otros ensayos, Madrid, Tecnos, 1986)

Quine, W.V., [1960] Word and Object, Cambridge, Massachusetts, The M.I.T. Press (las citas corresponden a la versión castellana Palabra y Objeto, Barcelona, Ed. Labor, 1968) 